\title{
Construção e validação de um índice de vulnerabilidade socioambiental para a vigilância e gestão de desastres naturais no Estado do Rio de Janeiro, Brasil
}

\author{
Construction and validation of a socio-environmental vulnerability \\ index for monitoring and management of natural disasters \\ in the state of Rio de Janeiro, Brazil
}

\author{
Raphael Mendonça Guimarães ${ }^{1}$ \\ Maíra Lopes Mazoto ${ }^{1}$ \\ Raphael Nascimento Martins ${ }^{2}$ \\ Cleber Nascimento do Carmo ${ }^{3}$ \\ Carmen Ildes Fróes Asmus ${ }^{1}$
}

${ }^{1}$ Instituto de Estudos em Saúde Coletiva, Universidade Federal do Rio de Janeiro. Av. Horácio Macedo S/N, Cidade Universitária. 21.944-970 Rio de Janeiro RJ Brasil. raphael@iesc.ufrj.br ${ }^{2}$ Instituto Brasileiro de Geografia e Estatística. ${ }^{3}$ Universidade Federal do Rio de Janeiro, campus Macaé.

\begin{abstract}
Floods account for approximately 40\% of natural disasters that occur around the world and they are therefore considered a major public health problem. While floods constitute a global problem, data from the International Strategy for Disaster Reduction showed that almost all of the deaths or individuals affected are concentrated in developing countries. It is assumed that, although they have natural causes, the consequences of floods also involve social issues. To try to predict such vulnerability in the occurrence of natural disasters, a social and environmental index that shows the degree of vulnerability of a location was developed in this paper. This index was developed using multivariate analysis involving factor anal$y$ sis and demographic, social and environmental variables. The index was applied in the municipalities of the state of Rio de Janeiro and compared with the official figures of the Civil Defense Unit. The results found suggest that the proposed index meets the expectation of predicting the vulnerability of the local population.
\end{abstract}

Key words Environmental health,Demography, Natural disasters, Vulnerability, Public health
Resumo As inundações representam aproximadamente $40 \%$ do total de desastres naturais ocorridos em todo o mundo, são consideradas, portanto, um importante problema de saúde pública. Embora as inundações constituam um problema global, dados da Estratégia Internacional de Redução de Desastres revelam que quase a totalidade dos óbitos ou afetados concentram-se em países em desenvolvimento. Presume-se que, embora tenham causas naturais, as consequências das inundações envolvem também questões sociais. Para tentar predizer o grau de vulnerabilidade de uma localidade na ocorrência de desastres naturais, foi desenvolvido neste trabalho um indice socioambiental. Para tanto, usou-se análise multivariada, envolvendo análise fatorial e variáveis demográficas, sociais e ambientais. O indice foi aplicado nos municípios do estado do Rio de Janeiro e comparado aos números oficiais da Defesa Civil. Os resultados encontrados sugerem que $o$ indice proposto atende a expectativa de predizer a vulnerabilidade da população local.

Palavras-chave Saúde ambiental, Demografia, Desastres naturais, Vulnerabilidade, Saúde pública 


\section{Introdução}

As inundações são o desastre natural mais comum em países desenvolvidos e em desenvolvimento, representando aproximadamente 40\% do total de desastres naturais ocorridos em todo o mundo. São consideradas, portanto, um importante problema de saúde pública ${ }^{1,2}$. Há uma tendência de aumento do número de pessoas expostas e afetadas por estes eventos no mundo. Porém, embora as inundações constituam um problema global, dados da Estratégia Internacional de Redução de Desastres revelam que 96\% da população exposta a estes eventos no mundo e 95\% dos óbitos registrados no ano de 2009 encontram-se concentrados nos países com renda per capita menor do que 3.705 dólares por ano ${ }^{3}$.

Diversas causas podem estar associadas à ocorrência de inundações, entre elas: as mudanças climáticas, o aquecimento global, chuvas intensas e localizadas, descarte inadequado de lixo, intensificação da agricultura, construção de barragens e hidrelétricas, desmatamento e erosão do solo ${ }^{4}$.

As inundações podem acarretar consequências ambientais: contaminação biológica e/ ou química da água para consumo humano e alimentos e alteração nos ciclos dos vetores, hospedeiros e reservatórios de doenças e nas formas de exposições ambientais dos humanos; consequências sobre a infraestrutura, serviços e economia local: interrupção do atendimento de saúde, efeitos sobre a agricultura e pecuária atingindo a produção e qualidade dos alimentos; consequências sobre a saúde humana: diarreias, gastroenterites, cólera, febre tifoide, leptospirose, desnutrição, transtornos mentais, conjuntivites, pressão arterial alta, dermatites e erupções cutâneas, infecções pulmonares, asmas, infecções renais, quedas, afogamentos, choques elétricos, traumas, entre outros'.

Quando falamos em danos humanos, aproximadamente 28.600 pessoas foram afetadas por inundações no Brasil e 1.100 morreram vítimas do evento no período de 1991 a 2010. Entre os afetados, incluem-se aqueles que foram vítimas fatais de inundações, desaparecidos ou desabrigados ${ }^{5}$.

A inundação brusca é um evento natural e recorrente no Estado do Rio de Janeiro, particularmente em função do volume de chuvas de verão. No entanto, a ação antrópica, a morfologia e as características regionais podem influenciar na extensão e intensidade do evento. Foram registradas 262 ocorrências de inundações no estado do Rio de Janeiro no período de 1991 a 2010. Quase todos os municípios do Estado (83\%) foram atingidos por essa tipologia de desastre, totalizando 76 municípios. Os municípios mais afetados, com recorrências entre 9-10 registros, foram: Angra dos Reis e Barra do Piraí, localizados na Mesorregião Sul Fluminense; Campos de Goytacazes e Macaé, no Norte Fluminense; Bom Jesus de Itabapoana, no Noroeste Fluminense; e Belford Roxo, na Mesorregião Metropolitana do Rio de Janeiro 5 .

Os desastres naturais podem ocorrer em qualquer continente ou país do mundo. Entretanto, existem regiões mais afetadas devido à magnitude e à frequência dos fenômenos e da vulnerabilidade do sistema social. Dados sobre desastres naturais no mundo revelam um maior impacto dos mesmos nos países subdesenvolvidos ou em desenvolvimento. No entanto, a gravidade deste impacto dependerá das condições de vulnerabilidade da comunidade ou sociedade atingida ${ }^{3}$.

As condições de vulnerabilidade são resultado de processos sociais e mudanças ambientais, denominada vulnerabilidade socioambiental. A vulnerabilidade socioambiental combina os processos sociais relacionados à precariedade das condições de vida da população (trabalho, saúde, renda, educação, habitações saudáveis, saneamento, etc.), que tornam determinados grupos populacionais (mulheres, crianças e idosos, por exemplo) mais vulneráveis aos desastres naturais com as mudanças ambientais resultantes da degradação ambiental (desmatamento de encostas e leitos de rios, por exemplo) $)^{3}$.

Diante deste contexto, os objetivos do presente estudo foram desenvolver e validar um indicador composto para predição de vulnerabilidade na ocorrência de desastres naturais.

\section{Materiais e métodos}

O estudo foi dividido em duas etapas: análise fatorial para definição do indicador composto e análise espacial do desempenho dos municípios com o índice criado.

\section{Definição do Indicador Composto}

Para a formulação do indicador composto, denominado Índice de Vulnerabilidade Socioambiental (IVSA), os municípios foram classificados utilizando-se de procedimentos de análise multivariada, envolvendo análise fatorial em componentes principais. Os fatores ou componentes principais $\left(\mathrm{F}_{\mathrm{i}}\right)$ são independentes, ou seja, 
ortogonais, determinados a partir de uma transformação linear das variáveis iniciais $\mathrm{X}_{\mathrm{i}}$, de acordo com o seguinte modelo matemático ${ }^{6}$ :

$$
X_{i}=a_{i 1} F_{1}+a_{i 2} F_{2}+a_{i 3} F_{3}+\ldots+\mathrm{A}_{i k} \mathrm{~F}_{k}+e_{i}
$$

Cada uma das " $k$ " variáveis observadas é descrita linearmente em termos das " $k$ " componentes não correlacionadas $\left(\mathrm{F}_{\mathrm{i}}\right) ;$ “ $\mathrm{a}_{\mathrm{i}}$ ” são os pesos ou cargas fatoriais que compõem a combinação linear, e " $\mathrm{e}_{\mathrm{i}}$ " corresponde ao erro. As cargas fatoriais " $a_{i k}$ " expressam os coeficientes de correlação entre cada uma das variáveis e seus respectivos fatores. Em cada fator, as variáveis mais representativas são aquelas cujas cargas fatoriais são maiores $\left(\mathrm{a}_{\mathrm{ik}}>0,30\right)$; quando a carga fatorial apresenta sinal negativo, significa influência negativa dessa variável no fator ${ }^{6,7}$.

O objetivo da análise fatorial é definir o relacionamento entre as variáveis de modo simples, utilizando menor número de variáveis do que o número original, reduzindo a dimensionalidade de um conjunto de variáveis por meio da utilização de suas intercorrelações. Em outras palavras, procura-se observar um conjunto de variáveis não observáveis a partir de um conjunto de variáveis observáveis ${ }^{8,9}$. Neste estudo, o conjunto de informações compunham 21 variáveis, a partir das quais pretendia-se construir um indicador composto.

O teste Kaiser-Meyer-Olkin (KMO) foi usado para avaliar a adequação do emprego da análise fatorial, considerando-se o conjunto de dados analisados ${ }^{10}$. O KMO é considerado adequado quando for superior a 0,5. A carga fatorial considerada foi acima de 0,3 para contribuir significativamente para o fator, após a rotação ortogonal pelo método varimax.

\section{Estruturação do Índice de Vulnerabilidade Socioambiental (IVSA)}

Para construir o Índice de Vulnerabilidade Socioambiental (IVSA) foram usados indicadores sociodemográficos dos municípios fluminenses, fazendo-se uso da propriedade de ortogonalidade associada aos escores fatoriais estimados. Com este procedimento foi testado se os escores fatoriais são ortogonais e observou-se a matriz de variância e covariância entre os mesmos. A matriz encontrada deve ser uma identidade para que os escores fatoriais sejam ortogonais.

Presumiu-se que os escores associados aos municípios tenham tido distribuição simétrica em torno da média zero. Desta forma, uma me- tade dos escores fatoriais teve sinais positivos enquanto a outra teve sinais negativos. Os municípios que apresentaram os menores IV obtiveram escores fatoriais negativos. Com o objetivo de evitar que altos escores fatoriais negativos elevem a magnitude dos índices associados a estes municípios, originou-se a seguinte transformação nos escores fatoriais tendo por objetivo trazer todos eles para o primeiro quadrante:

$$
f_{i j}=\frac{f-f_{\min }}{f_{\text {max }}-f_{\min }}
$$

na qual $f_{\min }$ e $f_{\max }$ são os valores mínimo e máximo observados para os escores fatoriais associados aos municípios, estabelecendo com este processo que todos os escores fatoriais estarão contidos no intervalo fechado entre zero e um.

Sendo assim, o IV foi estimado pela seguinte equação:

$$
I V_{i}=\left(f_{j 1}^{2}+f_{j 2}^{2}+f_{j 3}^{2}+\ldots+f_{j n}\right)^{1 / 2}
$$

onde $\mathrm{IV}_{\mathrm{i}}$ é o índice de vulnerabilidade associado ao i-ésimo município fluminense. Esperase que todos os coeficientes associados aos escores fatoriais relevantes sejam positivos.

As variáveis que entram na construção do IV estão listadas no Quadro 1.

Desta forma, constrói-se um Índice de Vulnerabilidade Socioambiental que não representa o percentual de vulneráveis. Este índice serve apenas para fazer hierarquia, tal como faz o Índice de Desenvolvimento Humano (IDH), por exemplo.

\section{Análise Espacial}

As unidades de análise foram os municípios do Estado do Rio de Janeiro, situado na Região Sudeste do Brasil, e que está dividido em 92 municípios, possui uma população de 15.383 .407 habitantes e uma área total de $43.696 .054 \mathrm{~km}^{2}$. Foi criado um mapa considerando a totalidade dos municípios, através da base cartográfica, referente ao ano de 2010. Foi avaliada a distribuição dos escores fatoriais de cada escore para cada município, e realizou-se em seguida o mapeamento do Índice de Vulnerabilidade Socioambiental (IVSA) ${ }^{11}$.

A seguir, foram mapeados os casos de vítimas de desastres naturais no Estado em 2010. Compreende-se por vítima como sendo todo aquele desabrigado, desaparecido ou morto em decorrência do desastre, usando-se os relatórios da Defesa Civil. O mapeamento realizado foi feito através dos tercis de distribuição dos escores fatoriais e das ocorrências de vítimas. 


\begin{tabular}{|c|c|c|}
\hline \multirow[t]{18}{*}{ 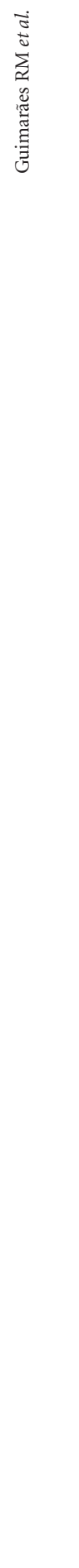 } & Quadro 1. Variáveis sele & ionadas para modelagem. \\
\hline & Variável & Definição \\
\hline & $\mathrm{HR}<15$ anos & Proporção de homens com idade inferior a 15 anos no meio rural \\
\hline & $\mathrm{MR}<15$ anos & Proporção de mulheres com idade inferior a 15 anos no meio rural \\
\hline & $\mathrm{HR} \geq 60$ anos & Proporção de homens com idade igual ou maior a 60 anos no meio rural \\
\hline & $\mathrm{MR} \geq 60$ anos & Proporção de mulheres com idade igual ou maior a 60 anos no meio rural \\
\hline & Proporção de Crianças & Proporção da população com menos de 5 anos \\
\hline & Proporção de Idosos & Proporção da população com mais de 60 anos \\
\hline & IDH renda & $\begin{array}{l}\text { Índice de Desenvolvimento Humano, componente renda: A renda é calculada tendo } \\
\text { como base o PIB per capita (por pessoa) do país. }\end{array}$ \\
\hline & IDH longevidade & $\begin{array}{l}\text { Indice de Desenvolvimento Humano, componente longevidade: avaliado considerando } \\
\text { a expectativa de vida ao nascer. }\end{array}$ \\
\hline & IDH educação & $\begin{array}{l}\text { Índice de Desenvolvimento Humano, componente educação: Para avaliar a dimensão } \\
\text { da educação o cálculo do IDH considera dois indicadores. O primeiro é a taxa de } \\
\text { alfabetização de pessoas com quinze anos ou mais de idade O segundo indicador } \\
\text { é a taxa de escolarização: somatório das pessoas, independentemente da idade, } \\
\text { matriculadas em algum curso, seja ele fundamental, médio ou superior, dividido pelo } \\
\text { total de pessoas entre } 7 \text { e } 22 \text { anos da localidade. }\end{array}$ \\
\hline & População $<1 / 2$ SM & $\begin{array}{l}\text { Número de pessoas (adultos e crianças) que vive com renda familiar inferior a 1/2 } \\
\text { salário mínimo }\end{array}$ \\
\hline & Renda média & Renda média per capita por habitante \\
\hline & Índice de Gini & $\begin{array}{l}\text { Grau de concentração da distribuição de renda domiciliar per capita de uma } \\
\text { determinada população e em um determinado espaço geográfico. }\end{array}$ \\
\hline & Razão de Renda & $\begin{array}{l}\text { Número de vezes que a renda agregada do quinto superior da distribuição da renda } \\
\text { (20\% mais ricos) é maior do que a renda do quinto inferior (20\% mais pobres) na } \\
\text { população residente }\end{array}$ \\
\hline & $\%$ pavimentação & Proporção do município com pavimentação regular das ruas \\
\hline & \% esgoto a céu aberto & Proporção do município com esgoto a céu aberto \\
\hline & $\%$ lixo acumulado & Proporção do município com lixo acumulado em ruas \\
\hline
\end{tabular}

Os dados utilizados na pesquisa provêm do censo demográfico de 2010 do $\mathrm{IBGE}^{11}$. Para a Análise Fatorial, utilizou-se o pacote estatístico SPSS, versão 19.0. O processamento e mapeamento dos dados foram feitos através do programa de código aberto TabWin, desenvolvido pelo Datasus.

\section{Resultados}

Foram aplicadas técnicas de análise fatorial em componentes principais e de agrupamento, ao conjunto de 16 variáveis socioambientais dos municípios do Rio de Janeiro, correspondentes ao ano de 2010. A partir da análise de correlação aplicada às variáveis de qualidade das águas, observou-se que a maioria destas está forte- 
mente correlacionada, com alta significância ( $\mathrm{p}$ $>0,001)$. Observou-se um valor para o teste de KMO de 0,75, o que significa que a análise fatorial obteve uma modelagem adequada. A análise de componentes principais revelou que 92\% do total da variância do modelo foram explicados por seis fatores, sendo estes identificados a partir dos critérios de Kaiser e pelos autovalores (Tabela 1).
A rotação varimax apresentou cargas fatoriais mais elevadas no primeiro fator constituído pelas variáveis a) Homens que vivem em ambiente rural com menos de 15 anos, b) Mulheres que vivem em ambiente rural com menos de 15 anos, c) Homens que vivem em ambiente rural com mais de 60 anos e d) Mulheres que vivem em ambiente rural com mais de 60 anos. Devido à natureza das variáveis compondo este fator, o

Tabela 1. Carga fatorial obtida pela rotação varimax.

\begin{tabular}{|c|c|c|c|}
\hline Variáveis & $\begin{array}{l}\text { População } \\
\text { Rural } \\
\text { Vulnerável }\end{array}$ & $\begin{array}{c}\text { Desenvolvimento } \\
\text { Humano }\end{array}$ & $\begin{array}{c}\text { Desigualdade } \\
\text { social }\end{array}$ \\
\hline Homens que vivem em ambiente rural com menos de 15 anos & 0,970 & & \\
\hline Mulheres que vivem em ambiente rural com menos de 15 anos & 0,959 & & \\
\hline Homens que vivem em ambiente rural com mais de 60 anos & 0,969 & & \\
\hline Mulheres que vivem em ambiente rural com mais de 60 anos & 0,965 & & \\
\hline \multicolumn{4}{|l|}{ Proporção de Crianças com até 5 anos } \\
\hline \multicolumn{4}{|l|}{ Proporção de Idosos com mais de 60 anos } \\
\hline IDH renda & & $-0,930$ & \\
\hline IDH longevidade & & $-0,957$ & \\
\hline IDH escolaridade & & $-0,952$ & \\
\hline \multicolumn{4}{|l|}{ Proporção da população que vive com menos de $1 / 2 \mathrm{SM} /$ mês } \\
\hline \multicolumn{4}{|l|}{ Renda Média per capita } \\
\hline Índice de Gini & & & 0,932 \\
\hline Razão de Renda & & & 0,943 \\
\hline \multicolumn{4}{|l|}{ \% de ruas com pavimentação } \\
\hline \multicolumn{4}{|l|}{$\%$ de casas com cobertura regular de esgoto } \\
\hline \multicolumn{4}{|l|}{$\%$ de casas com coleta regular de lixo } \\
\hline \% variância explicada & 26,29 & 17,54 & 13,71 \\
\hline$\%$ acumulada & 26,29 & 43,83 & 57,54 \\
\hline \multirow[t]{2}{*}{ Variáveis } & Planejamento & Vulnerabilidade $\mathrm{V}$ & Vulnerabilidade \\
\hline & Urbano & Demográfica & Econômica \\
\hline \multicolumn{4}{|l|}{ Homens que vivem em ambiente rural com menos de 15 anos } \\
\hline \multicolumn{4}{|l|}{ Mulheres que vivem em ambiente rural com menos de 15 anos } \\
\hline \multicolumn{4}{|l|}{ Homens que vivem em ambiente rural com mais de 60 anos } \\
\hline \multicolumn{4}{|l|}{ Mulheres que vivem em ambiente rural com mais de 60 anos } \\
\hline Proporção de Crianças com até 5 anos & & 0,914 & \\
\hline Proporção de Idosos com mais de 60 anos & & 0,916 & \\
\hline \multicolumn{4}{|l|}{ IDH renda } \\
\hline \multicolumn{4}{|l|}{ IDH longevidade } \\
\hline \multicolumn{4}{|l|}{ IDH escolaridade } \\
\hline Proporção da população que vive com menos de $1 / 2 \mathrm{SM} /$ mês & & & 0,838 \\
\hline Renda Média per capita & & & 0,689 \\
\hline \multicolumn{4}{|l|}{ Índice de Gini } \\
\hline \multicolumn{4}{|l|}{ Razão de Renda } \\
\hline \% de ruas com pavimentação & $-0,751$ & & \\
\hline$\%$ de casas com cobertura regular de esgoto & 0,914 & & \\
\hline$\%$ de casas com coleta regular de lixo & 0,790 & & \\
\hline \% variância explicada & 13,46 & 11,27 & 10,35 \\
\hline$\%$ acumulada & 71,00 & 82,27 & 92,62 \\
\hline
\end{tabular}

Legenda: IDH - Índice de Desenvolvimento Humano; SM - Salário Mínimo. 
qual explicou 26,29\% da variância dos dados, ele foi denominado População Rural Vulnerável.

O segundo fator, responsável por $17,54 \%$ da variância, foi composto pelas variáveis e) componente Renda do IDH, f) componente Longevidade do IDH e g) componente Educação do IDH, recebendo o nome de Desenvolvimento Humano.

Contabilizando $13,71 \%$ da variância, o terceiro fator incluiu as variáveis $\mathrm{h}$ ) Índice de Gini e i) Razão de Renda, sendo denominado de Desigualdade Social.

O quarto fator incluído no modelo, que contribuiu com 13,46\% da variância deste, é composto por j) percentual de ruas com pavimentação, k) percentual de casas com cobertura regular de esgoto e 1) percentual de casas com coleta regular de lixo, sendo denominado como Planejamento Urbano.

O quinto fator, que contribuiu com $11,27 \%$ da variância, incluiu as variáveis m) Proporção de Crianças com até 5 anos e n) Proporção de Idosos com mais de 60 anos e foi denominado Vulnerabilidade Demográfica.

Finalmente, o sexto fator, denominado Vulnerabilidade Econômica, foi composto pelas variáveis o) Proporção da população que vive com menos de $1 / 2 \mathrm{SM} / \mathrm{mês}$ e p) Renda Média per capita, explicando um total de 10,35\% da variância.

Em seguida, com os fatores realizou-se a transformação nos escores fatoriais, conforme apresentado na fórmula 2, de forma a evitar que altos escores fatoriais negativos elevem a magnitude dos índices associados aos municípios. Finalmente, procedeu-se o cálculo final do Índice, conforme descrito na fórmula 3.

A partir disto, realizou-se o mapeamento dos municípios de acordo com o valor do Índice de Vulnerabilidade Socioambiental (Figura 1a). Para ambos, os escores fatoriais e os índices foram divididos em tercis.

Finalmente, os dados referentes ao número de vítimas por desastres naturais foram mapeados com o auxílio do TabWin (Figura 1b).

Ao observar simultaneamente as Figuras 1a e $1 b$, verifica-se que em todos os municípios fluminenses enquadrados no terceiro tercil do IV correspondem ao terceiro tercil do mapa que representa o número de afetados pelos alagamentos no Estado do Rio de Janeiro, segundo os dados da defesa civil para o ano de 2010. A mesma correspondência pode ser verificada ao se analisar os municípios do primeiro tercil em ambos os mapas.

Não há exceções, em todas as regiões do estado Fluminense: Metropolitana, Noroeste Fluminense, Norte Fluminense, Baixadas Litorâneas, Serrana, Centro-Sul Fluminense, Médio Paraíba e Costa Verde, o padrão de concentração de número de afetados e a aplicação de IV correspondem.

Este resultado sugere fortemente que o Índice de Vulnerabilidade Socioambiental é um bom

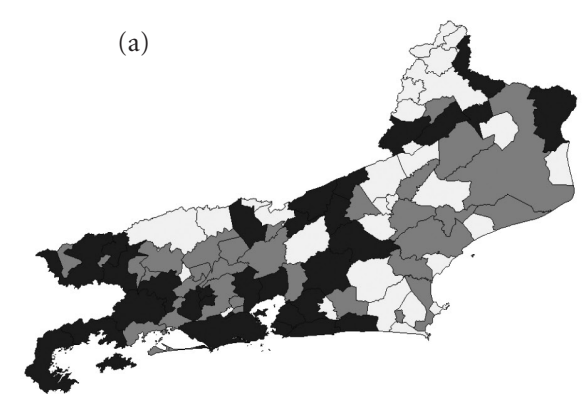

Primeiro Tercil (b)

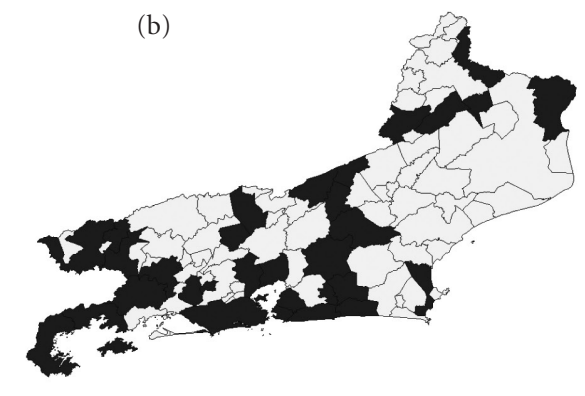

Segundo Tercil

Figura 1. Distribuição Espacial dos Tercis do Índice de Vulnerabilidade Socioambiental (a) e do número de afetados por enchentes (b) no Estado do Rio de Janeiro, 2010.

Nota: O mapa de afetados mostra que a quantidade de municípios com nenhum afetado por enchente notificados pelo AVADAN representou mais de $60 \%$ dos municípios do estado, tendo portanto agregado o primeiro e segundo tercis. 
parâmetro para predição de vulnerabilidade na ocorrência de desastres naturais.

\section{Discussão}

A vulnerabilidade socioambiental resulta de estruturas socioeconômicas que produzem, simultaneamente, condições de vida precárias e ambientes deteriorados, expressando-se também como menor capacidade de redução de riscos e baixa resiliência ${ }^{3}$.

Estudos similares a este na área de vulnerabilidade social/socioambiental a desastres naturais foram feitos no Brasil e no mundo. Ainda não há um consenso de quais variáveis devem ser utilizadas. Porém, todos os trabalhos analisados convergem para a ideia de que a vulnerabilidade deve ser estudada conjuntamente nos âmbitos naturais e socioeconômicos ${ }^{12}$.

Licco"13, no seu trabalho "Vulnerabilidade social e desastres naturais: uma análise preliminar sobre Petrópolis, Rio de Janeiro", afirma que quando se analisa a vulnerabilidade de uma comunidade é fundamental que os fatores humanos sejam considerados, uma vez que são eles que, de forma geral, mais influenciam na severidade de um desastre. Dentre esses fatores destacam-se: Riqueza, Educação, Governança, Tecnologia, Idade e Gênero. Segundo o autor, estes fatores importam de várias maneiras. A riqueza é importante, pois pobres são menos capazes de pagar por moradias e outras infraestruturas que podem suportar eventos extremos. A educação é importante porque indivíduos com maior nível de educação tendem a aprender a evitar e/ou reduzir muitos impactos em relação a indivíduos menos educados. Governança porque governos podem promover políticas que reduzem vulnerabilidades e estabelecer estruturas administrativas encarregadas dessa redução. Tecnologia, porque, por exemplo, pode melhorar a capacidade de prever eventos extremos. Idade e gênero porque crianças, idosos e mulheres tendem a ser mais vulneráveis por possuírem menos força física.

Cutter et al. ${ }^{14}$ em Georgetown County, South Carolina, EUA, afirmam que perdas oriundas de riscos ambientais têm aumentado na última década, levando a uma reorientação dos sistemas de gestão de emergência que estão superando a lógica de simples resposta a eventos ocorridos. Há uma notável mudança na política, com mais ênfase na redução de perdas através de mitigação, preparação. Ainda segundo os autores do artigo, o grau em que as populações são vulneráveis aos riscos, no entanto, não é unicamente dependente da proximidade da fonte da ameaça ou da natureza física dos fatores de risco, as variáveis sociais, também desempenham um papel importante na determinação da vulnerabilidade. O artigo apresenta, portanto, um método para a avaliação da vulnerabilidade em termos espaciais utilizando ambos os indicadores biofísicos e sociais, a saber: população total; total de unidades habitacionais; número de mulheres; número de residentes não brancos; número de pessoas com menos de 18 anos; número de pessoas com mais de 65 anos; valor principal da casa; número de casas móveis.

Rygel et al. ${ }^{15}$, cujo trabalho teve como objetivo demonstrar um método de agregação de indicadores de vulnerabilidade que resulta em um índice composto deste, mas que evita os problemas associados à atribuição de pesos, utilizou uma técnica baseada no ranking de Pareto para um complexo, paisagem socioeconômica desenvolvida, exposição a tempestades associadas a furacões. Os indicadores de vulnerabilidade social para este perigo são desenvolvidos e uma análise de componentes principais é realizada em proxies para estes indicadores. A Vulnerabilidade social geral é calculada através da aplicação de classificação de Pareto para esses componentes principais. Nesse trabalho ele utilizou como variáveis pobreza, gênero, raça, idade e inabilidades físicas ou mentais.

$\mathrm{Na}$ Inglaterra, Tapsell et al. ${ }^{16}$ apresentam resultados de pesquisa sobre os impactos que as inundações podem ter sobre as pessoas afetadas, complementando, assim, os dados existentes sobre as perdas monetárias que podem ocorrer em eventos de inundação. No artigo em questão é criado um índice de vulnerabilidade social a inundações que utiliza como método o índice Towsend e usa as seguintes variáveis: taxa de desemprego, densidade demográfica; casas sem carro; \% de famílias sem casa própria; número de residentes com doenças crônicas; número de pessoas com mais de 65 anos; pais/mães solteiros (as).

$\mathrm{Na}$ Europa, a pesquisa de Balica et al. ${ }^{17}$ foi composta por dados categorizados em Componente Social: densidade populacional, população em área de risco, população em situação de pobreza, população na área urbana, população na área rural, população com deficiência; Componente Econômico: uso da terra, proximidade do rio, proximidade da área de inundação; Componente Ambiental: uso da terra, área degradada, tipo de vegetação; e, Componente Físico: topografia, chuvas, duração da inundação, proximidade do rio, velocidade de fluxo, umidade do solo, etc. 
O trabalho de Kuhlicke et al. ${ }^{18}$, realizado na Alemanha, Itália e Reino Unido, apresenta os resultados de avaliações de vulnerabilidade sociais realizados em diferentes estudos de caso das inundações na Europa. Uma constatação compartilhada entre os estudos de caso é que não foi possível identificar um conjunto comum de indicadores socioeconômicos e demográficos para explicar a vulnerabilidade social de grupos e/ou indivíduos para todas as fases dos eventos desastrosos. Da mesma forma, verificou-se que os indicadores de localização e de eventos específicos não tinham a relevância que se esperava que eles tivessem. Os resultados reforçam que a vulnerabilidade é um produto de contextos socioeconômicos e demográficos, culturais e institucionais espaciais específicos que impõem não só desafios específicos às tentativas de avaliar a vulnerabilidade social em geral. Os estudos de caso deste artigo utilizaram como variáveis: Variáveis socioeconômicas e demográficas: idade, gênero, emprego, ocupação, nível de escolaridade, deficiências, composição familiar, renda familiar, ter casa própria ou não, tamanho da residência; Variáveis de redes sociais: ter Corpo de Bombeiros e/ou Defesa Civil ou não, nível de confiança nas autoridades locais.

A pesquisa de Freitas e Cunha ${ }^{19}$ teve como objetivo principal apresentar um estudo de modelagem de dados que tem como resultado o mapa de vulnerabilidade socioambiental de 17 concelhos da Região Centro de Portugal. São apresentadas as caracterizações dos concelhos em estudo, como subsídios para as análises de vulnerabilidade realizadas. A metodologia adotada para a análise da vulnerabilidade baseou-se em Análise Fatorial que agregou variáveis sociais e ambientais das seguintes naturezas: Economia e Condição de Vida, População, Saúde e Proteção Social, Condição de Alojamento e Edifícios,
Condições Territoriais e Ambientais, Educação, Justiça, Agricultura.

Finalmente, no Brasil, no estado do Paraná, Furlan et al. ${ }^{20}$ utilizaram dados de Fragilidade física: Declividade, Drenagem, Ocorrência dos eventos; Fragilidade socioeconômica: Densidade populacional, Percentagem de população urbana e rural, Faixas etárias, Educação e Índice de GINI; Falta de resiliência: PIB per capita, Número de estabelecimentos de saúde, Razão de sexo, Razão de dependência. Há inúmeros outros exemplos que endossam esta abordagem.

Como pode ser visto nos exemplos citados, pesquisas europeias, brasileiras e estadunidenses têm sido elaboradas, agregando dados sociais e ambientais no intuito de predizer a vulnerabilidade socioambiental.

\section{Conclusão}

Pode-se concluir, portanto, que as inundações registradas no Estado do Rio de Janeiro são resultado de uma combinação da ocorrência de eventos extremos com as condições de vulnerabilidade do local. Assim, faz-se necessário o conhecimento espacial das áreas mais vulneráveis à ocorrência destes eventos, visando subsidiar a elaboração de planos de preparação e resposta para o enfrentamento destes eventos e consequente minimização dos seus riscos e impactos.

A utilização de variáveis sociais, socioeconômicas e demográficas, em conjunto com variáveis ambientais parecem, portanto, uma combinação que tende a predizer com algum nível de acurácia as situações de vulnerabilidade socioambiental e devem ser levadas em consideração pelos institutos que lidam com essas situações problemáticas no intuito de diminuírem os impactos das inundações.

\section{Colaboradores}

RM Guimarães participou da concepção do estudo, análise dos dados, redação e revisão final do manuscrito; ML Mazoto e RN Martins participaram da redação e revisão final do manuscrito; CN Carmo e CIF Asmus participaram da revisão final do manuscrito. 


\section{Referências}

1. Torti J. Flood in Southeast Asia: A health priority. Journal of Global Health 2012; 2(2):1-6.

2. Eripidou E, Murray V. Public Health impacts of floods and chemical contamination. J Public Health 2004; 26(4):376-383.

3. Freitas CM, Carvalho ML, Ximenes EF, Arraes EF, Gomes JO. Vulnerabilidade socioambiental, redução de riscos de desastres e construção da resiliência - lições do terremoto no Haiti e das chuvas fortes na região Serrana, Brasil. Cien Saude Colet 2012; 17(6):3689-3696.

4. Freitas CM, Ximenes EF. Enchentes e saúde pública: uma questão na literatura científica recente das causas, consequências e respostas para prevenção e mitigação. Cien Saude Colet 2012; 17(6):1601-1615.

5. Centro Universitário de Estudos e Pesquisas Sobre Desastres (Ceped UFSC). Volume Rio de Janeiro. In: Ceped UFSC. Atlas Brasileiro de Desastres Naturais. Florianópolis: Ceped UFSC; 2011. p. 1-63.

6. Alcântara VS. Vulnerabilidade socioambiental na Macrorregião da Costa Verde [dissertação]. Rio de Janeiro: Escola Nacional de Ciências Estatísticas; 2012.

7. Bouroche JM, Saporta G. Análise de dados. Rio de Janeiro: Zahar Editores; 1980

8. Mingoti SA. Análise de dados através de métodos de estatística multivariada: uma abordagem prática. $2^{\text {a }}$ ed. Belo Horizonte: UFMG; 2013.

9. Peduzzi P, Dao H, Herold C, Mouton P. Assessing global exposure and vulnerability towards natural hazards: the disaster risk index. Natural Hazards and Earth System Sciences 2009; 9:1149-1159.

10. Hair Junior JF, Anderson RE, Tatham RL, Black WC. Análise multivariada de dados. $5^{\mathrm{a}}$ ed. Porto Alegre: Bookman; 2005.

11. Instituto Brasileiro de Estatística e Geografia (IBGE). Atlas do Censo Demográfico de 2010. Rio de Janeiro: IBGE; 2013.

12. Gall M. Indices of Social Vulnerability to Natural Hazards: A Comparative Evaluation. Los Angeles: University of South California; 2007.

13. Licco EA. Vulnerabilidade social e desastres naturais: uma análise preliminar sobre Petrópolis, Rio de Janeiro. Rev. de Saúde, Meio Ambiente e Sustentabilidade 2013; 8(1):25-41.
14. Cutter SL, Mitchell JT, Scott MS. Revealing the vulnerability of people and places: A case study of Georgetown County, South Carolina. Annals of the Association of American Geographers 2000; 90(4):713-737.

15. Rygel L, O'sullivan D, Yarnal B. A method for constructing a social vulnerability index: an application to hurricane storm surges in a developed country. Mitigation and Adaption Strategies for Global Change 2006; 11:741-764.

16. Tapsell SM, Penning-Rowsell EC, Tunstall SM, Wilson TL. Vulnerability to flooding: health and social dimensions. Philosophical Transactions of the Royal Society 2002; 360(1796):1511-1525.

17. Balica SF, Douben N, Wright NG. Flood Vulnerability Indices at Varying Spatial Scales. Water Science \& Technology 2009; 60(10):2571-2580.

18. Kuhlicke C, Scolobig A, Tapsell S, Steinfuhrer A, Marchi B. Contextualizing social vulnerability: findings from case studies across Europe. Natural Hazards 2011; 58(2):789-810.

19. Freitas MI, Cunha L. Modelagem de dados socioambientais visando estudos de vulnerabilidade: $\mathrm{O}$ caso de 17 concelhos do centro de Portugal. Revista Geonorte 2012; 1(4):816-829.

20. Furlan CM, Lacruz MSP, Sausen TM. Vulnerabilidade socioeconômica à ocorrência de eventos extremos: proposta metodológica. In: Anais do XV Simpósio Brasileiro De Sensoriamento Remoto - SBSR; 2011; São José dos Campos. p. 4540-4546.

Artigo apresentado em 23/06/2014

Aprovado em 11/07/2014

Versão final apresentada em 15/07/2014 